\title{
Generalized Huberman-Rudnick scaling law and robustness of $q$-Gaussian probability distributions
}

\author{
Ozgur Afsar ${ }^{1, *}$ and Ugur Tirnakli ${ }^{1,2,+}$ \\ ${ }^{1}$ Department of Physics, Faculty of Science, \\ Ege University, 35100 Izmir, Turkey \\ ${ }^{2}$ Division of Statistical Mechanics and Complexity, \\ Institute of Theoretical and Applied Physics (ITAP) \\ Kaygiseki Mevkii, 48740 Turunc, Mugla, Turkey
}

(Dated: January 1, 2018)

\begin{abstract}
We generalize Huberman-Rudnick universal scaling law for all periodic windows of the logistic map and show the robustness of $q$-Gaussian probability distributions in the vicinity of chaos threshold. Our scaling relation is universal for the self-similar windows of the map which exhibit period-doubling subharmonic bifurcations. Using this generalized scaling argument, for all periodic windows, as chaos threshold is approached, a developing convergence to $q$-Gaussian is numerically obtained both in the central regions and tails of the probability distributions of sums of iterates.
\end{abstract}

*Electronic address: ozgur.afsar@ege.edu.tr

${ }^{\dagger}$ Electronic address: ugur.tirnakli@ege.edu.tr 


\section{INTRODUCTION}

It is well-known that many complex systems exhibit transitions from periodic motion to chaos through period doubling route like Rayleigh-Benard system in a box [1], forced pendulum [2], logistic map [3], Chirikov map [4] etc. The logistic map, defined as

$$
x_{t+1}=1-a x_{t}^{2}
$$

(where $0<a \leq 2$ is the control parameter and the phase space $x_{t}$ is between $[-1,1]$ with $t=0,1,2, \ldots)$, is a good example to observe Feigenbaum route generated by pitchfork bifurcations and its universal features. This map has its critical point, denoted by $a_{c}$, at $a_{c}=1.401155189 \ldots$, which can be approached from left (i.e., from periodic region) via period doubling procedure where $2^{\infty}$ periods accumulate at this critical point usually described as chaos threshold. This point can also be approached from right (i.e., from chaotic region) via band merging procedure where infinite number of bands merge at the critical point [5]. A sketchy view of these approaches from left and from right to $a_{c}$ is given in Fig. 1 a. In the chaotic region $\left(a>a_{c}\right)$, there exist many windows of higher periodic cascades of $s 2^{n}$ periods, where $s$ is an integer and $n$ is the degree of period-doublings. It is worth noting that, within each window, reverse bifurcations of $s 2^{n+1}$ bands merging into $s 2^{n}$ bands can easily be detected although the width of these windows decreases rapidly as long as $s \neq 1$. As an example, $s=3$ case (namely, period 3 case) is illustrated in Fig. 1b.

This self-similar structure of the map is of sensible importance and leads Feigenbaum to develop a scaling theory for the non-chaotic period-doubling region of the bifurcations, which enables one to localize the control parameter value at the bifurcation from $2^{n}$ period to $2^{n+1}$ via scaling relation

$$
\left|a-a_{c}\right| \sim \delta^{-n}
$$

where $\delta=4.699 \ldots$ is the Feigenbaum constant [6]. On the other hand, it is possible to obtain Eq. (2) from the famous Huberman-Rudnick scaling law [7], which shows that the envelope of the Lyapunov exponents near $a_{c}$ exhibits a universal behavior, similar to that of an order parameter close to the critical point of phase transition. This relation can be written as

$$
\lambda=\lambda_{0}\left|a-a_{c}\right|^{\nu}
$$



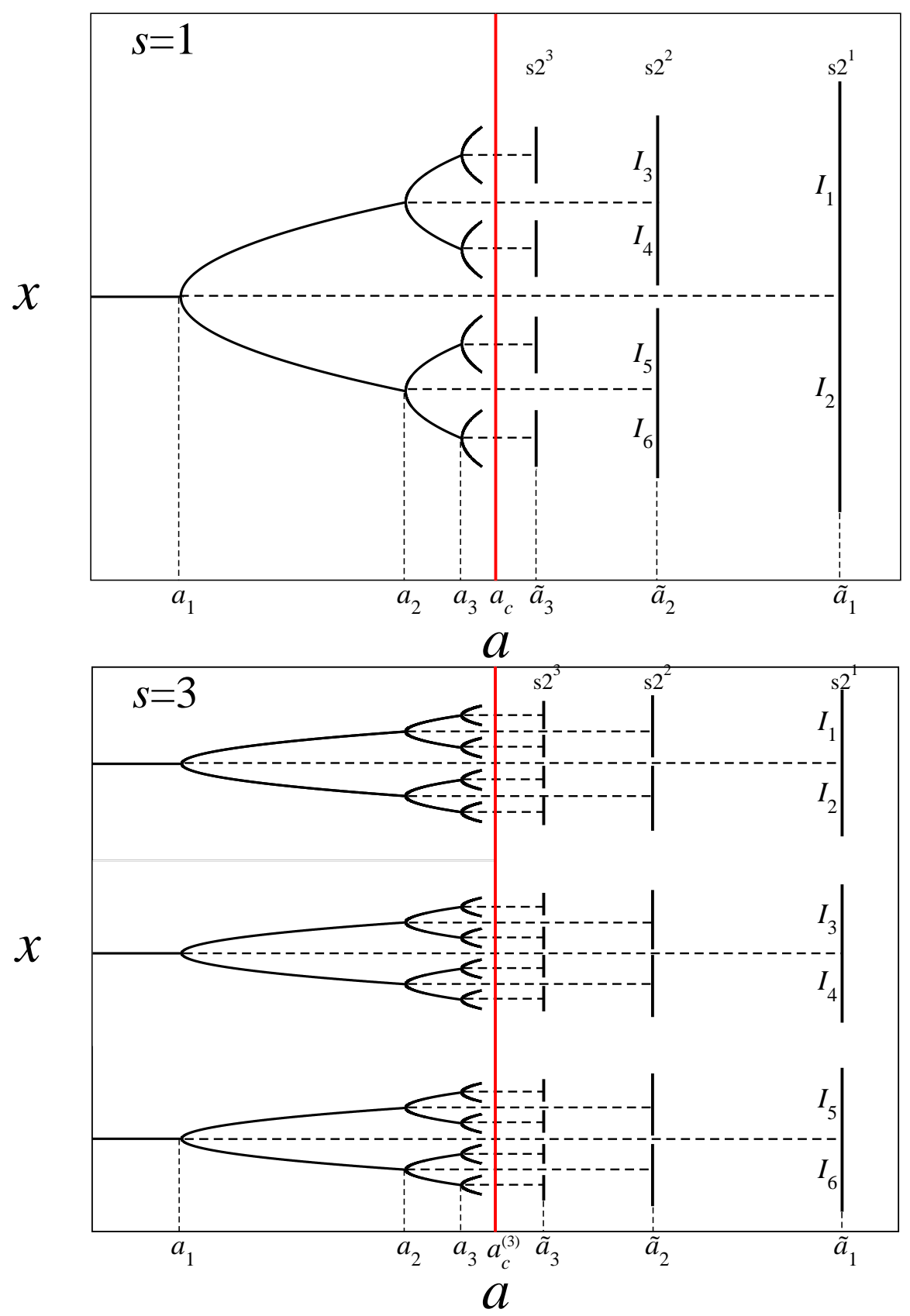

FIG. 1: (a) Sketchy view of the standard bifurcation diagram of the logistic map. Approaching chaos threshold $\left(a_{c}=1.401155189 \ldots\right)$ from left via period-doubling route is evident with bifurcation points denoted as $a_{n}(n=1,2, \ldots, \infty)$. Corresponding band merging approach to $a_{c}$ is depicted with merging points denoted as $\tilde{a}_{n}$ at each of which $2^{n}$ bands appear. (b) Sketchy view of period 3 window in the chaotic region of the logistic map. Approaching chaos threshold $\left(a_{c}^{(3)}=1.779818075 \ldots\right)$ of this periodic window from left via period-doubling route is evident with bifurcation points denoted as $a_{n}$. Corresponding band merging approach to $a_{c}^{(3)}$ is depicted with merging points denoted as $\tilde{a}_{n}$ at each of which $3 \times 2^{n}$ bands appear. 
where $a>a_{c}, \nu=\ln 2 / \ln \delta, \lambda$ is the Lyapunov exponent and $\lambda_{0}$ is a constant. Eq. (2) can easily be obtained from Eq. (3). For a values slightly above the chaos threshold, there exist $2^{n}(n=1,2, \ldots, \infty)$ chaotic bands, which approach the Feigenbaum attractor as $n \rightarrow \infty$ by the band splitting procedure. In that region, if we start from two trajectories separated by a distance $d_{0}$ within one of these chaotic bands, the separation of trajectories increases exponentially. If the trajectories start off in one band, after $2^{n}$ iterations they will be back in the original band. Then they will be separated by the amount

$$
d_{2^{n}}=d_{0} e^{\lambda 2^{n}}=d_{0} e^{\lambda_{0}}
$$

where $\lambda_{0}=\lambda 2^{n}$ is the effective Lyapunov exponent (a constant value) [7]. Substituting the effective Lyapunov exponent into Eq. (3) immediately gives

$$
2^{-n}=\left|a-a_{c}\right|^{\ln 2 / \ln \delta},
$$

from where Eq. (2) is easily obtained.

This scaling relation, in fact, is exactly the one used in [8], where the probability distributions of the sums of the iterates of the logistic map, as $a_{c}$ is approached from the band merging region, have been shown to be well approached by $q$-Gaussians provided that the appropriate number of iterations $\left(N^{*}\right)$ is obtained from the above-mentioned scaling relation.

$q$-Gaussians, defined as,

$$
P(y)= \begin{cases}P(0)\left[1-\beta(1-q) y^{2}\right]^{\frac{1}{1-q}} & \text { for } \beta(1-q) y^{2}<1 \\ 0 & \text { otherwise }\end{cases}
$$

(where $q<3$ and $\beta>0$ are parameters and the latter controls the width of the distribution) are the distributions that optimize, under appropriate constraints, the nonadditive entropy $S_{q}$ (defined to be $\left.S_{q} \equiv\left(1-\sum_{i} p_{i}^{q}\right) /(q-1)\right)$, on which nonextensive statistical mechanics is based [9, 10]. As $q \rightarrow 1$, $q$-Gaussians recover the Gaussian distribution.

Although in Nature many stochastic processes, consist of sum of many independent or nearly independent variables, are known to converge Gaussian distribution due to the standard central limit theorem [11, 12], in recent years several complex systems such as lowdimensional dissipative maps in the vicinity of chaos threshold [13 15], high dimensional dissipative systems [16] and conservative maps [17, 18] are shown to exhibit probability distributions that are well approached by $q$-Gaussians. 
Our main aim in this paper is two-folded: firstly, we try to generalize the HubermanRudnick scaling law to all periodic windows of the logistic map, secondly, using this generalized version of the Huberman-Rudnick scaling law, we analyse the robustness of the probability distribution of the sums of iterates of the logistic map as chaos threshold is approached.

\section{GENERALIZATION OF THE HUBERMAN-RUDNICK SCALING LAW}

In order to find generalized version of the Huberman-Rudnik scaling law, let us start by denoting the accumulation point of a particular periodic cycle $s$ as $a_{c}^{(s)}$. For example, $a_{c}^{(3)}$ and $a_{c}^{(5)}$ stand for the accumulation points of period 3 and period 5 windows inside the chaotic region. These points can be found as $a_{c}^{(3)}=1.779818075 \ldots$ and $a_{c}^{(5)}=1.631019835 \ldots$. Then, one need to check whether the form of the Huberman-Rudnick scaling law is valid for all other periodic cycles in the chaotic region. More precisely, one need to check whether the exponent $\nu$ in the scaling law is equal to $\ln 2 / \ln \delta$ as in the standard case. Therefore, we have first checked this and found that, for all periodic windows, the envelope of the Lyapunov exponents, in $\log \lambda$ vs $\log \left(a-a_{c}\right)$ plot, is given by a slope 0.449 , which is nothing but $\ln 2 / \ln \delta$. Hence we can now write the Huberman-Rudnick scaling law for all periodic cycles as

$$
\lambda=\lambda_{0}\left[a-a_{c}^{(s)}\right]^{\ln 2 / \ln \delta} .
$$

At this point, it should be recalled that, for a particular period $s$ window, a trajectory that starts in one band will be back in the same band after $s 2^{n}(n=1,2, \ldots, \infty)$ iterations. If we use this feature in the definition of the effective Lyapunov exponent, namely $\lambda_{0}=\lambda s 2^{n}$, then one can write the Huberman-Rudnick scaling law for other periodic windows as

$$
\lambda=\lambda s 2^{n}\left[a-a_{c}^{(s)}\right]^{\ln 2 / \ln \delta}
$$

and

$$
2^{-n}=s\left|a-a_{c}^{(s)}\right|^{\ln 2 / \ln \delta} .
$$

This equation enables us to obtain the generalized Huberman-Rudnick scaling law as 


$$
\left|a-a_{c}^{(s)}\right|=\delta^{-n-\frac{\ln s}{\ln 2}} .
$$

This new scaling relation is valid for all periodic windows including the standard case for $s=1$, which immediately recovers the standard scaling law given in Eq. (22).

\section{ROBUSTNESS OF PROBABILITY DISTRIBUTIONS}

Now let us concentrate on the probability distributions of the sums of iterates of the logistic map, which can be written as

$$
y:=\sum_{i=1}^{N}\left(x_{i}-\langle x\rangle\right),
$$

where $x_{i}$ are the iterates of the logistic map and $x_{1}$ is the initial value regarded as a random variable. It has analytically been proved that, for strongly chaotic systems, the probability distribution of $y$ becomes Gaussian for $N \rightarrow \infty[19,20]$. Here, the average $\langle\ldots\rangle$ is calculated as time average. On the other hand, as mentioned before in Section I, several complex systems of the type low and high dimensional dissipative and conservative exist where the probability distribution does not approach to Gaussian, and therefore violating the standard central limit theorem due to possible lack of ergodicity and mixing properties. For such systems, it is necessary to take the average over not only a large number of $N$ iterations but also a large number of $M$ randomly chosen initial values, namely,

$$
\langle x\rangle=\frac{1}{M} \frac{1}{N} \sum_{j=1}^{M} \sum_{i=1}^{N} x_{i}^{(j)} .
$$

As chaos threshold is approached, the logistic map has already been studied in this respect [8, 13, 21, 22]. As it has already been argued in [8], in principle, in order to attain chaos threshold point exactly (i.e., approaching this point with infinite precision), one needs to take $n \rightarrow \infty$, which, in other words, means that the necessary number of iterations to achieve the limit distribution at the chaos threshold is $N^{*} \rightarrow \infty$ since $N^{*}=2^{2 n}$. Since this is, no doubt, unattainable in any numerical experiment, one can only approach to this critical point using the appropriate values for $\left(a, N^{*}\right)$ pairs coming from the Huberman-Rudnick scaling law. As long as this scaling law is obeyed, developing $q$-Gaussian shape of the limit distribution, as chaos threshold is approached, has been clearly shown in [8]. On the way of approaching 
chaos threshold, for any approximation level of finite $\left(a, N^{*}\right)$ pairs, if the number of iterations used is too much larger than $N^{*}$ and therefore violating the Huberman-Rudnick scaling law (i.e., $N>>N^{*}$ ), it is of course not surprising that the probability distribution starts to approach to Gaussian form from its central part since the system starts to feel that it is not exactly at the chaos threshold. Such numerical examples can be found in [8, 21]. On the other hand, if the number of iterations used is too much smaller than $N^{*}$ and therefore violating again the Huberman-Rudnick scaling law (i.e., $N<<N^{*}$ ), then the summation starts to be inadequate to approach the shape of the limit probability distribution and it exhibits a kind of peaked or multifractal distribution. Such numerical examples have already been given in [13, 21, 22].

In the remainder of this work, we try to provide further evidence on the robustness of the $q$-Gaussian probability distributions seen as the chaos threshold is approached. In order to accomplish this task, we investigate other periodic windows (numerically chosen examples are period 3 and 5) making use of our generalized Huberman-Rudnick law.

As separated band structure for periodic cycle 2 goes from $2^{1}$ to $2^{\infty}$ with $2^{n}(n=$ $1,2, \ldots, \infty)$, for any periodic cycle $s$, the same behavior would be to go from $s 2^{0}$ to $s 2^{\infty}$ with $s 2^{k}(k=0,1, \ldots, \infty)$. It is evident that there is $k \rightarrow(n-1)$ transformations between $k$ and $n$ mathematically. Generically, for any periodic cycle $s$ in the chaotic region, one needs to perform $s 2^{k}$ iterations of the map for a given initial value with a control parameter $a$ obtained from the generalized scaling law. After $s 2^{k}$ iterations, the system will basically fall into the same band of the band splitting structure. This means that the sum of the iterates $\sum_{i=1}^{s 2^{k}} x_{i}$ will essentially approach to a fixed value $w=s 2^{k}\langle x\rangle$ plus a small correction $\Delta w_{1}$ which describes the small fluctuations of the position of the $s 2^{k}$ th iterate within the chaotic band. Hence, one can write

$$
y_{1}=\sum_{i=1}^{s 2^{k}}\left(x_{i}-\langle x\rangle\right)=\Delta w_{1} .
$$

If we continue to iterate for another $s 2^{k}$ times, we obtain

$$
y_{2}=\sum_{i=s 2^{k}+1}^{2 s 2^{k}}\left(x_{i}-\langle x\rangle\right)=\Delta w_{2} .
$$

The new fluctuation $\Delta w_{2}$ is not expected to be independent from the old one $\Delta w_{1}$, since correlations of iterates decay very slowly if we are close to the critical point. Continuing 
this $2^{k}$ times, we finally obtain

$$
y_{2^{k}}=\sum_{i=s 2^{2 k}-s 2^{k}+1}^{s 2^{2 k}}\left(x_{i}-\langle x\rangle\right)=\Delta w_{2^{k}}
$$

if we iterate the map $s 2^{2 k}$ times in total. The total sum of iterates

$$
y=\sum_{i=1}^{s 2^{2 k}}\left(x_{i}-\langle x\rangle\right)=\sum_{j=1}^{2^{k}} \Delta w_{j}
$$

can thus be regarded as a sum of $2^{k}$ random variables $\Delta w_{j}$, each being influenced by the structure of the $s 2^{k}$ chaotic bands at distance $a-a_{c}^{(s)}=\delta^{-n-\frac{\ln s}{\ln 2}}$ from the Feigenbaum attractor. At this distance to chaos threshold, in order to see the limit distribution, appropriate number of iterations would be $N^{*}=s 2^{2 k}$, which corresponds to $N^{*}=s 2^{2 n-2}$ after $k \rightarrow(n-1)$ transformation.

Now we are ready to check the shape of the probability distribution of any periodic windows obeying our generalized Huberman-Rudnick scaling law. Chosen examples of possible periodic windows are period 3 and period 5 since they are the largest two periodic windows available in the chaotic region. Although conceptually nothing is changed for small sized windows, numerical analysis is getting more difficult as windows sizes are decreasing. Numerically used values are given in the Table for our period 3 and 5 analysis. Control parameter values $a$ are chosen so that the precision of corresponding $n$ values, coming from the generalized Huberman-Rudnick scaling law as

$$
n=-\frac{\ln \left|a-a_{c}^{(s)}\right|}{\ln \delta}-\frac{\ln s}{\ln 2},
$$

would be the same (see Table). This means that we are approaching the critical point with $a$ values located on a straight line with a given slope. Our results are given in Fig. 2 for period 3 and in Fig. 3 for period 5. In both cases four representative points systematically approaching the chaos threshold is given. It is clear from Fig. 2a and Fig. 3a that the probability distributions of both periodic windows approach to a $q$-Gaussian. It is also evident that, as the chaos threshold is better approached, the tails of the distribution develops better on the $q$-Gaussian, signaling that the limit distribution obtained at the exact chaos threshold point would be a $q$-Gaussian with infinitely long tails. We also present the same data in Fig. $2 \mathrm{~b}$ and Fig. 3b in a different way so that a straight line would be expected for $q$-Gaussians. 

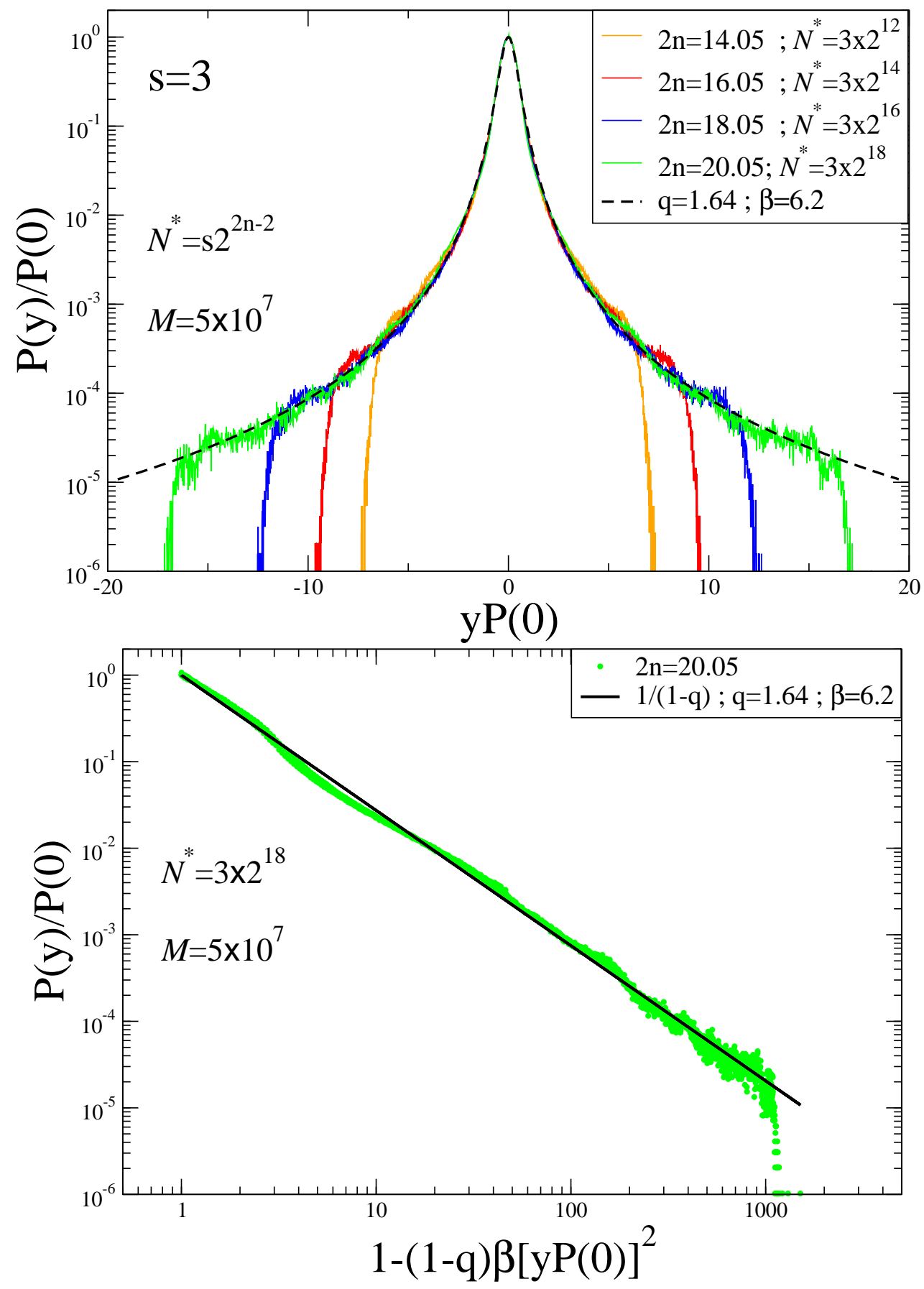

FIG. 2: (a) Probability distribution functions of period $3(s=3)$ for four representative cases with different $n$ values. As $n$ increases, numerical convergence to a $q$-Gaussian with $q=1.63$ and $\beta=6.3$ is appreciated. (b) The same distribution plotted against $1-(1-q) \beta[y P(0)]^{2}$ on a $\log -\log$ plot for the case which is the closest to the chaos threshold. A straight line is expected with a slope $1 /(1-q)$ if the curve is a $q$-Gaussian. It is clearly seen how the straight line is surrounded by the log-periodically modulated curves. 

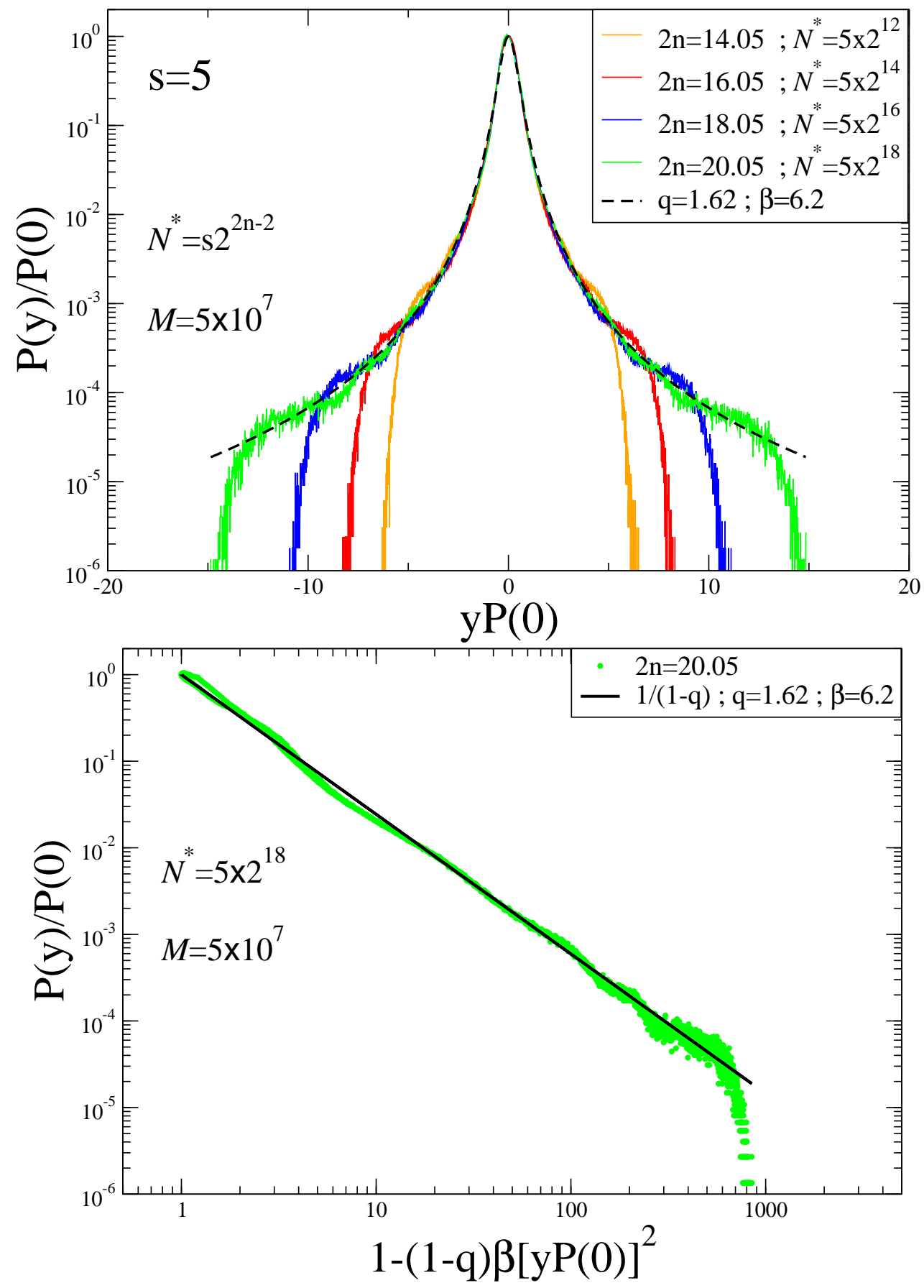

FIG. 3: (a) Probability distribution functions of period $5(s=5)$ for four representative cases with different $n$ values. As $n$ increases, numerical convergence to a $q$-Gaussian with $q=1.62$ and $\beta=6.3$ is appreciated. (b) The same distribution plotted against $1-(1-q) \beta[y P(0)]^{2}$ on a $\log -\log$ plot for the case which is the closest to the chaos threshold. A straight line is expected with a slope $1 /(1-q)$ if the curve is a $q$-Gaussian. It is clearly seen how the straight line is surrounded by the log-periodically modulated curves. 
Only the closest cases to the chaos threshold for each periodic window are plotted. It is seen from these plots that the curves develop on top of a straight line surrounded by log-periodic modulations.

TABLE I: Parameter values used in this work. The values of $n$ obtained from the generalized scaling law using Eq. (17), the corresponding $N^{*}$ values and the values of $q$ and $\beta$ (estimated from simulations) are listed. $s=1$ case, already discussed in [8], has also been included in the Table for comparison.

\begin{tabular}{|c|c|c|c|c|c|}
\hline$s$ & $a$ & $2 n$ & $N^{*}$ & $q$ & $\beta$ \\
\hline \multirow{4}{*}{1} & 1.401588 & 10.05 & $2^{10}$ & \multirow{4}{*}{1.70} & \multirow{4}{*}{6.2} \\
\hline & 1.401248 & 12.05 & $2^{12}$ & & \\
\hline & 1.401175 & 14.05 & $2^{14}$ & & \\
\hline & 1.40115945 & 16.05 & $2^{16}$ & & \\
\hline \multirow{4}{*}{3} & 1.779819805038384 & 14.05 & $3 \times 2^{12}$ & \multirow{4}{*}{1.64} & \multirow{4}{*}{6.2} \\
\hline & 1.779818446177396 & 16.05 & $3 \times 2^{14}$ & & \\
\hline & 1.779818155150985 & 18.05 & $3 \times 2^{16}$ & & \\
\hline & 1.779818092822039 & 20.05 & $3 \times 2^{18}$ & & \\
\hline \multirow{4}{*}{5} & 1.63102039110464 & 14.05 & $5 \times 2^{12}$ & \multirow{4}{*}{1.62} & \multirow{4}{*}{6.2} \\
\hline & 1.63101995463619 & 16.05 & $5 \times 2^{14}$ & & \\
\hline & 1.63101986115802 & 18.05 & $5 \times 2^{16}$ & & \\
\hline & 1.63101984113785 & 20.05 & $5 \times 2^{18}$ & & \\
\hline
\end{tabular}

$\dagger$ Values related to $s=1$ case are taken directly from the Table given in ref. [8].

\section{CONCLUSIONS}

Our main results obtained in this paper can be summarized as follows: (i) For the logistic map having self-similar structure, Huberman-Rudnick universal scaling law has been generalized, which becomes now consistent to all periodic windows in the chaotic region of the map. This new generalized scaling law is of sensible importance since it enables us to produce 
self-similar structure of the map and to explain all band merging structures in all available periodic windows using only one generalized formula. (ii) The standard Huberman-Rudnick scaling law has already been used in [8, 23] and $q$-Gaussian probability distributions have been observed as the standard period 2 accumulation point is approached. However, in order to test the robustness of $q$-Gaussian distributions, a first straightforward attempt should be to analyse other critical points (chaos thresholds) of different periodic windows located in the chaotic region of the logistic map. Since the generalized Huberman-Rudnick scaling law obtained in the first part of this paper now enables us to localize appropriate $\left(n, N^{*}\right)$ pairs as the accumulation point is approached, we managed to check two representative periodic windows. For each case studied here (and possibly for all other periodic windows) it is numerically shown that the $q$-Gaussian probability distributions with log-periodic oscillations are again the observed distributions and developing better as the critical point becomes closer. These results clearly indicate the robustness of the $q$-Gaussian probability distributions seen in the vicinity of chaos threshold. Although the obtained $q$ values seem to exhibit a slow decreasing tendency as the size of the periodic window becomes smaller, we believe that the genuine limit distribution of the chaos threshold (for all $s$ values) would converge to a $q$-Gaussian with a unique $q$ value, which is expected to be in the interval $[1.6,1.75]$.

Finally it is worth mentioning that the results obtained here are expected to be valid for all other dissipative maps sharing the same universality class with the logistic map. As an open question that can be addressed in a future work, one can mention the analysis of appropriate scaling law for the systems exhibiting quasi-periodic route to chaos.

\section{Acknowlegments}

This work has been supported by TUBITAK (Turkish Agency) under the Research Project number $112 \mathrm{~T} 083$.

[1] V.L. Gertsberg and G.I. Sivashinsky, Prog. Theor. Phys. 66 (1981) 1219.

[2] D. D’Humieres, M.R. Beasley, B.A. Huberman, A. Libchaber, Phys. Rev. A 26 (1982) 3483.

[3] R. May, Nature 261 (1976) 45.

[4] B. V. Chirikov, Phys. Rep. 52 (1979) 263. 
[5] J. P. Crutchfield, J. D. Farmer and B. A. Huberman, Phys. Rep. 92 (1982) 45.

[6] M. Feigenbaum, J. Stat. Phys. 19 (1978) 25; 21 (1979) 669.

[7] B. A. Huberman and J. Rudnick, Phys Rev. Lett. 45 (1980) 154.

[8] U. Tirnakli, C. Tsallis and C. Beck, Phys. Rev. E 79 (2009) 056209.

[9] C. Tsallis, J. Stat. Phys. 52 (1988) 479; E.M.F. Curado and C. Tsallis, J. Phys. A 24 (1991) L69; Corrigenda: 24 (1991) 3187 and 25 (1992) 1019; C. Tsallis, R.S. Mendes and A.R. Plastino, Physica A 261 (1998) 534.

[10] C. Tsallis, Introduction to Nonextensive Statistical Mechanics - Approaching a Complex World (Springer, New York, 2009).

[11] N.G. van Kampen, Stochastic Processes in Physics and Chemistry (North Holland, Amsterdam, 1981).

[12] A.Ya. Khinchin, Mathematical Foundations of Statistical Mechanics (Dover, New York, 1949).

[13] U. Tirnakli, C. Beck and C. Tsallis, Phys. Rev. E 75 (2007) 040106(R).

[14] G. Ruiz and C. Tsallis, Eur. Phys. J. B 67 (2009) 577.

[15] O. Afsar and U. Tirnakli, Phys. Rev. E 82 (2010) 046210.

[16] G. Miritello, A. Pluchino and A. Rapisarda, Physica A 388 (2009) 4818.

[17] S. M. Duarte Queiros, Phys. Lett. A 373 (2009) 1514.

[18] G. Ruiz, T. Bountis and C. Tsallis, Int. J. Bifur. Chaos 22 (2012) 1250208.

[19] P. Billingsley, Convergence of Probability Measures (Wiley, New York, 1968).

[20] C. Beck, Physica A 169 (1990) 324.

[21] M.A. Fuentes and A. Robledo, J. Stat. Mech. (2010) P01001.

[22] P. Grassberger, Phys. Rev. E 79 (2009) 057201.

[23] C. Tsallis and U. Tirnakli, J. Phys.: Conf. Ser. 201 (2010) 012001. 